\title{
Digital Library Based on RSS Technology
}

\author{
Yihua Zhang \\ Chengdu University of Information Technology, Library, Chengdu 610225, China \\ zhyihu@cuit.edu.cn
}

Keywords: RSS; library; push service; information push system.

\begin{abstract}
In order to facilitate the user to select the information, a RSS information push system for library information was proposed. The actual situation of the library was investigated. Combined with the recommended technology, the library information push platform was designed. The design of the information platform included three parts: the reader, the generation of RSS feed and the management of user information. Readers used the platform to subscribe to the channel of interest. The information pushed by the library was automatically taken when needed. It was an online reader. The results showed that the system was feasible. It can provide more effective and personalized information services for readers. The system has some practical and application value for the library reader service.
\end{abstract}

\section{Introduction}

With the development of science and technology, computer technology and network technology have penetrated into every field of society. It fundamentally changes the way of work, study and life. Library has undergone fundamental changes. Nowadays, the library has developed from the traditional library to the digital library. The basic function of the library is to provide readers with more quick and convenient services and meet the needs of the readers through the organization and management of the literature. Using the means of educational technology, the library provides the reader with personalized service. Information push technology is born in this case [1].

Push technology provides a new information service model. In this model, the service is active. With the help of the network, the staff use the push technology to carry out information service for the users. The information in the resource base and the information on the Internet are collected and screened. According to the specific requirements of the user, the information is filtered and processed. It is a new type of personalized information value-added service [2]. At present, the information push technology, especially the channel push technology based on RSS, has been widely used in domestic large portals, such as Sina and Xinhuanet. As long as the user subscribes to the corresponding RSS channel, the client software will be able to continue to receive the specific channel information from the server side. The introduction of push technology changes the way of information acquisition. As a result, push services have become an effective means of attracting new users and consolidating old users [3]. Through the RSS push technology, it provides the reader with an efficient way to get the information quickly and meet the individual needs.

\section{Methodology}

\subsection{RSS Push Technology}

RSS (Really Simple Syndication) is based on the channel push service. It is called a really simple aggregation [4]. In general, the principle of RSS information push service provides RSS feed for content providers. According to the changes in the content, RSS feed is updated in real time. With the aid of the RSS reader, users add the URL address of the RSS feed to the reader and synchronize RSS feed at regular intervals. When the site content page is not opened, the user reads the summary first. Then, according to the needs, the relevant content is further read. A user can subscribe to the RSS feed of multiple sites, to complete aggregation of information from different sources. After subscribing, the new information will continue to be "pushed" in real time to the user. Users do not have to get information between multiple sites. The information flow of the RSS information push service is shown in Figure 1. 


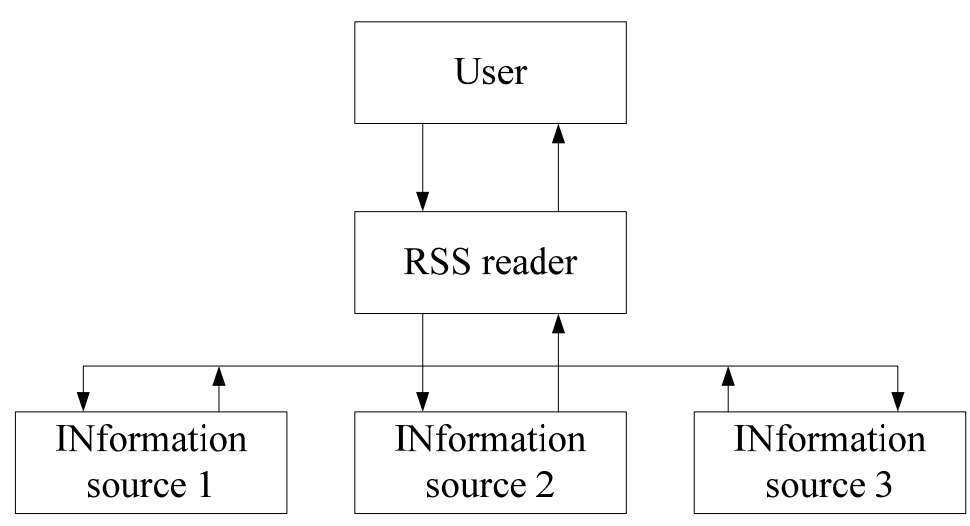

Figure. 1 RSS information push service

The RSS specification describes a simple subset of the XML element. It is committed to the establishment of standard and open channel description frameworks and content collection mechanisms, which do not define complex elements and attributes. On the basis of defining the core framework and basic element cables, the XML-namespace one mechanism is used to reuse other metadata sets to extend their own elements and functions. At present, the metadata set (named domain) directly reused in RSS is Dublin Core and Syndication.

RSS treats websites as a combination of a series of channels. Each channel also contains a series of channel resources. As a result, a description of the channel and the resources contained can be achieved for a web site as a resource collection. For each channel, it is usually described by name, address link, channel profile, and so on. Each information resource description includes information headlines, information address links, information profiles, and authors. In the channel, optional subelements such as Image and TextInput can also be used. RSS describes the web content file RSS feed by describing the channel and the included resources. RSS feed is published and promoted by the website of content providers. It is also used by the website of the content integrator (also known as "portal"), or is obtained by personal users through RSS aggregator software [5]. A brief RSS feed structure is as follows:

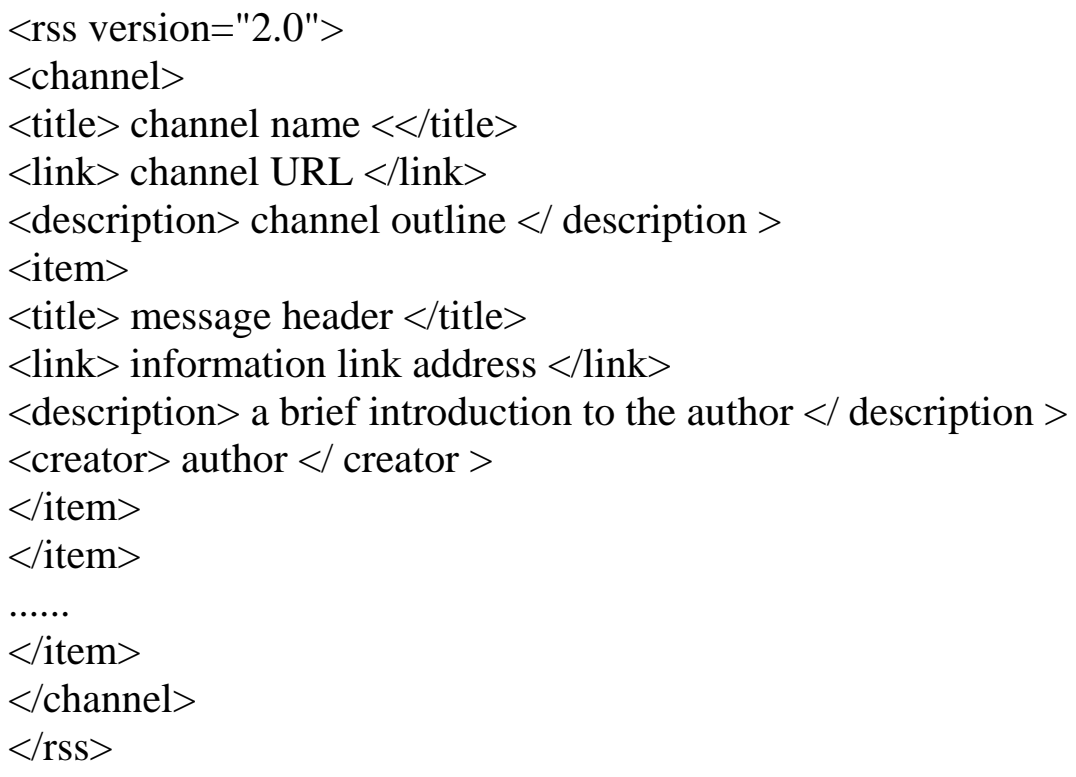

The key to developing RSS information push services is to provide personalized RSS feed for subscribers [6]. The RSS information push service saves the time and energy of the user, improves the efficiency of obtaining information, and ensures the timeliness of information. However, the meaning of the "push" technology of network content is not limited to the "real-time update" of the user - side information. It supports a new "information release platform" on the Internet. The online information release is no longer monopolized by a few. Every content provider, whether an enterprise or an individual, can use this platform to push information content to all users instead of being filtered and delayed by middlemen. It greatly expands the scope and content of Internet information, enriches 
the means of information acquisition, and provides wider space for the information sharing of the whole society. In addition, it provides a "aggregator" (RSS reader) for the receiver. The "aggregator" can subscribe to a variety of information sources. Users can manage the content of the Internet centrally, so as to ensure the comprehensive, timely and accurate information. The utilization of information has been improved. It also provides a new model for information services. The relevant information is collated and edited. The service is pushed in time. The relevant personnel pay attention to the latest industry trends and grasp the direction of professional development.

\subsection{Design of Information Service System Based on RSS Technology}

The designed RSS based information service system is a push information service platform that can be released on the home page of the library. The reader uses the platform to subscribe to the channel. The information pushed by the library is automatically obtained, which is an online reader. The design of the information platform consists of three parts. The first is the reader. The reader has the functions of user login, channel management subscriptions, unsubscribe, and information reading. The second is the generation of RSS feed (RSS source). The library will push the channel to generate the corresponding RSS feed for subscription. The third is the management of user information. This module can analyze the content of readers and subscriptions.

The basic principle of the system is designed according to the content setting of the library push service. Users can use web pages to read and do not have to install readers, which is convenient for readers to subscribe and use. It has a personalized interface and can be changed as needed. User information and data are calculated [7]. According to the above system design objectives, the topology diagram of the RSS based information system is designed as Figure 2.

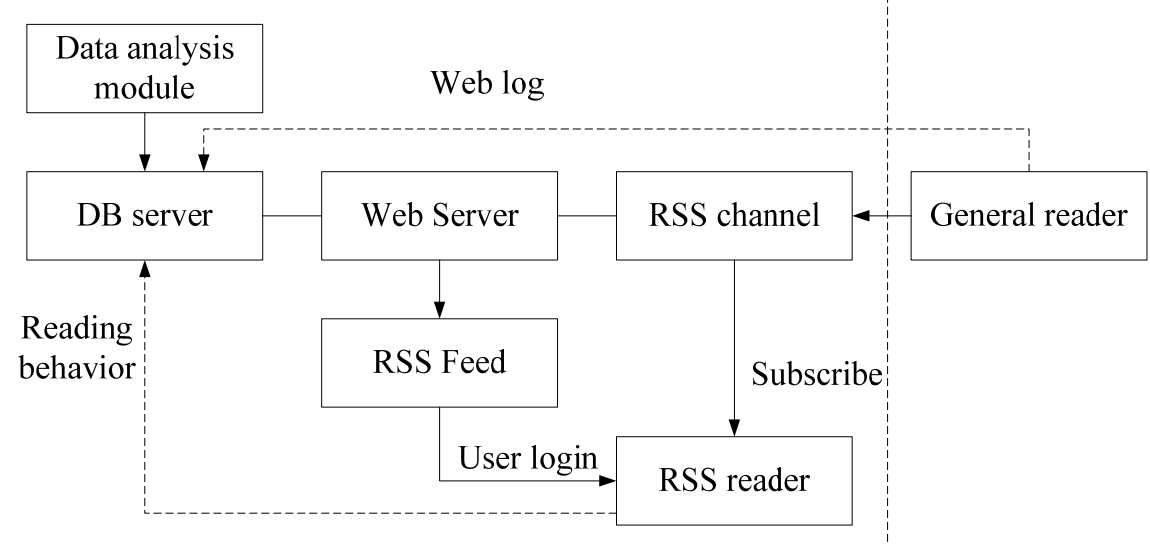

Figure. 2 Topology diagram of the design and implementation of information system

The login module can be managed by the administrator, and the readers of the school and the outside readers can be logged in. The user's login topology is shown in Figure 3.

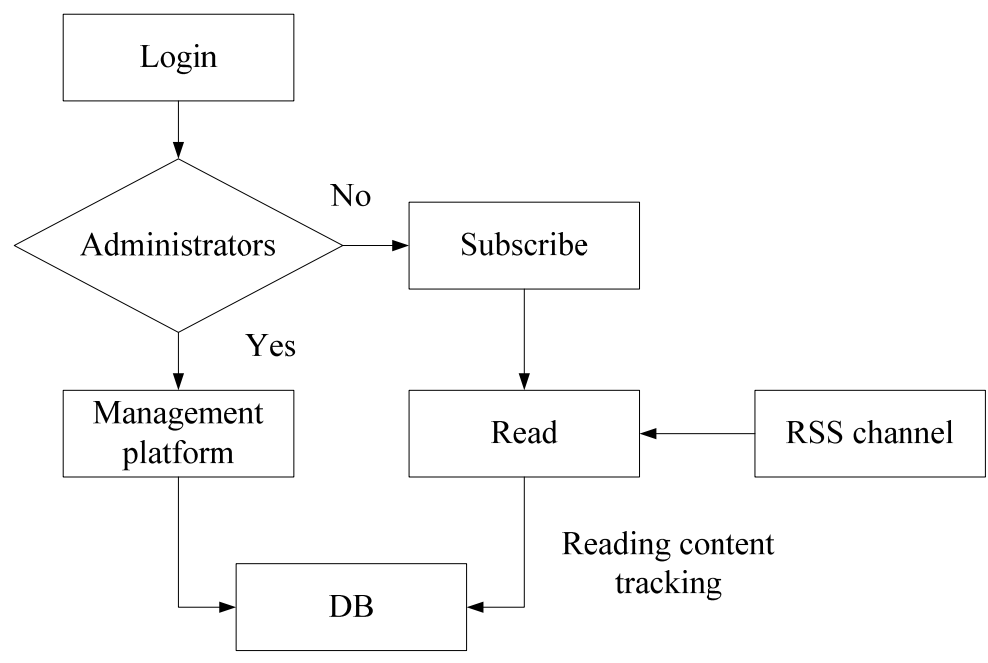

Figure. 3 The user's login topology 
All kinds of free readers on the Internet have been studied. The online free reader is powerful. However, it lacks the individualized function of management and statistics. The purpose of this reader's design is to read in the form of a web page without the need to install a reader and a plug-in. The main page concept is shown in Figure 4.

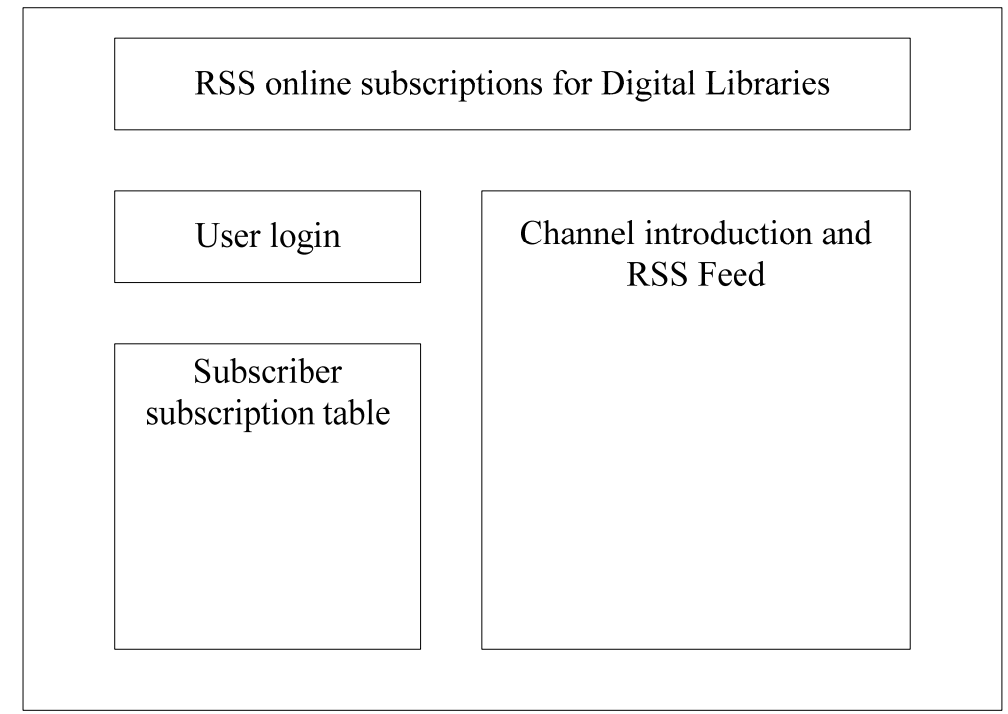

\subsection{The Generation of RSS Feed}

Figure. 4 Conception of the main page of a reader

Each library will design multiple push channels on the basis of the library. As an example, the method of designing and implementing the RSS feed is illustrated by the notification of the new book of the library. The library introduces each new book to the reader in a certain way. Readers can get to know the latest library books and find new books in time. The utilization of books has been improved. To this end, the library can design the RSS new book notification channel. Once a reader is subscribed, the reader can get the information in time [8]. It is realized by getting the relevant data of the new book first, and then generating the RSS feed.

RSS feed is automatically generated by the JAVA program. The following is a part of the core code that is implemented:

The data in a text file is read by line.

File f=new File("c: Inewbook.txt")

FileInputStream fis=new FileInputStream(f);

InputStreamReader isr=new InputStreamReader(fis);

BuferedReader br=nes BuferedReader(isr);

Based on the read data, each <item> content in the RSS feed is constructed.

while((sline=br.readLine())!=null)

\{using the substring () function to split each data, read out nominations, publish instructions, ISBN and so on to generate $<$ item $>$ content $\}$

Output RSS feed.

File f=new File("../nesbook.xml"); // Setting up an output XML file

FileOutputStream fis=new FileOutputStream Writer(fis);

BufferedWriter br=new BufferedWriter(isr);

Br.write(head+content+tail); //Writing a string to a file newbook.xml.

\section{Results and Discussion}

Under the development platform ultreadit editor and Dreamveaver2004, as well as the operation system Window XP and IIS system components, the library information push system based on RSS technology is implemented. The database design with statistical functions is implemented using Acess. It includes the reader's information database and the reader's use of the content record database. The implementation diagram of the system's main page is shown in Figure 5. 


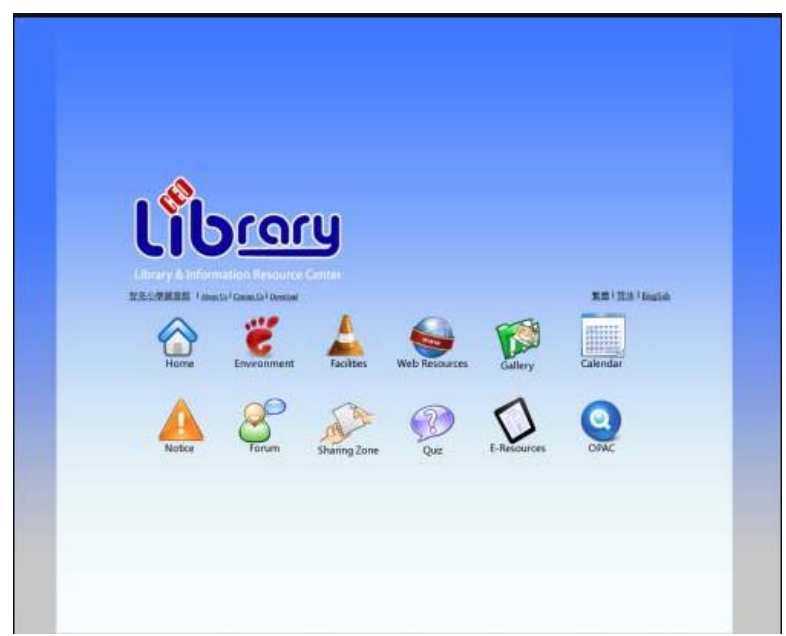

Figure. 5 The implementation of the system

\section{Conclusion}

Through the research of push technology, a library information push system based on RSS is designed. The system consists of three parts: the reader, the generation of RSS feed, and the management of user information. First of all, RSS technology is introduced in detail. Then, the design framework of the system, the user login topology, and the idea of the reader's main page are discussed. The core code of the RSS feed is explained. Finally, the system designed in this paper is realized under the Window XP system. The research shows that the information push system of the library is feasible. The digital library can provide the readers with effective and personalized information services. It has a certain practical value for the service work of the library readers.

\section{References}

[1]. Hua X, Si L, Zhuang X, et al. Investigations about new methods of library marketing in Chinese “985” Project Universities. Library Management, 2015 , 36 (6/7), pp.408-420.

[2]. Tarhini A, Hassouna M, Abbasi MS, et al. Towards the Acceptance of RSS to Support Learning: An Empirical Study to Validate the Technology Acceptance Model in Lebanon. Electronic Journal of e-Learning , 2015 , 13, pp. 30-41.

[3]. Chung KY, Na YJ, Lee JH. Interactive Design Recommendation Using Sensor Based Smart Wear and Weather WebBot. Wireless Personal Communications, 2013 , 73 (2), pp.243-256.

[4]. Adeniyi DA, Wei Z, Yongquan Y. Automated web usage data mining and recommendation system using K-Nearest Neighbor (KNN) classification method. Applied Computing \& Informatics, 2016,12 (1), pp. 90-108.

[5]. Wang X, Chen M. PreFeed: Cloud-Based Content Prefetching of Feed Subscriptions for Mobile Users. IEEE Systems Journal, 2014 , 8 (1), pp. 202-207.

[6]. Johnson SM, Osmond A, Holz RJ. Developing a current awareness service using really simple syndication (RSS). Journal of the Medical Library Association Jmla, 2009 , 97 (1), pp.52.

[7]. Ma D. Use of RSS feeds to push online content to users. Decision Support Systems, 2012 , 54 (1), pp.740-749.

[8]. Singh KP, Gill MS. Web 2.0 technologies in libraries: a survey of periodical literature published by Emerald. Library Review, 2013 , 62 (3), pp. 177-198(22). 